\title{
Genome-wide identification of the histone acetyltransferase gene family in Triticum aestivum
}

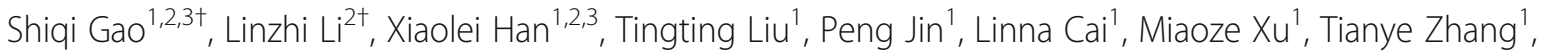
Fan Zhang ${ }^{1}$, Jianping Chen ${ }^{1}$, Jian Yang ${ }^{1 *}$ and Kaili Zhong ${ }^{1 *}$ (i)

\begin{abstract}
Background: Histone acetylation is a ubiquitous and reversible post-translational modification in eukaryotes and prokaryotes that is co-regulated by histone acetyltransferase (HAT) and histone deacetylase (HDAC). HAT activity is important for the modification of chromatin structure in eukaryotic cells, affecting gene transcription and thereby playing a crucial regulatory role in plant development. Comprehensive analyses of HAT genes have been performed in Arabidopsis thaliana, Oryza sativa, barley, grapes, tomato, litchi and Zea mays, but comparable identification and analyses have not been conducted in wheat (Triticum aestivum).

Results: In this study, 31 TaHATs were identified and divided into six groups with conserved gene structures and motif compositions. Phylogenetic analysis was performed to predict functional similarities between Arabidopsis thaliana, Oryza sativa and Triticum aestivum HAT genes. The TaHATs appeared to be regulated by cis-acting elements such as LTR and TC-rich repeats. The qRT-PCR analysis showed that the TaHATs were differentially expressed in multiple tissues. The TaHATs in expression also responded to temperature changes, and were all significantly upregulated after being infected by barley streak mosaic virus (BSMV), Chinese wheat mosaic virus (CWMV) and wheat yellow mosaic virus (WYMV).
\end{abstract}

Conclusions: These results suggest that TaHATs may have specific roles in the response to viral infection and provide a basis for further study of TaHAT functions in T. aestivum plant immunity.

Keywords: Histone acetyltransferases, Triticum aestivum, Genome-wide, Temperature, Wheat virus, Expression analysis

\footnotetext{
* Correspondence: nather2008@163.com; zhongkaili@nbu.edu.cn

'Shiqi Gao and Linzhi Li contributed equally to this work.

'State Key Laboratory for Managing Biotic and Chemical Threats to the

Quality and Safety of Agro-products, Key Laboratory of Biotechnology in

Plant Protection of Ministry of Agriculture and Zhejiang Province, Institute of

Plant Virology, Ningbo University, Ningbo 315211, China

Full list of author information is available at the end of the article
}

(c) The Author(s). 2021 Open Access This article is licensed under a Creative Commons Attribution 4.0 International License, which permits use, sharing, adaptation, distribution and reproduction in any medium or format, as long as you give appropriate credit to the original author(s) and the source, provide a link to the Creative Commons licence, and indicate if changes were made. The images or other third party material in this article are included in the article's Creative Commons licence, unless indicated otherwise in a credit line to the material. If material is not included in the article's Creative Commons licence and your intended use is not permitted by statutory regulation or exceeds the permitted use, you will need to obtain permission directly from the copyright holder. To view a copy of this licence, visit http://creativecommons.org/licenses/by/4.0/. The Creative Commons Public Domain Dedication waiver (http://creativecommons.org/publicdomain/zero/1.0/) applies to the data made available in this article, unless otherwise stated in a credit line to the data. 


\section{Background}

In eukaryotic cells, genomic DNA (gDNA) and histones are tightly packaged into a complex structure known as chromatin. Nucleosomes are the basic structural unit of chromatin: approximately 146 base pairs (bp) of DNA are wrapped around a histone octamer, which itself contains two molecules each of histones H2A, H2B, H3, and $\mathrm{H} 4$. Each histone contains a structured spherical domain and an unstructured N-terminal tail that extends from the core nucleosome $[1,2]$. These tails undergo a variety of posttranslational modifications, including acetylation, methylation, phosphorylation, ubiquitination, and ADPribosylation. Histone acetylation is a dynamic and reversible process that is co-regulated by histone acetyltransferase (HAT) and histone deacetylase (HDAC) [3]. HAT transfers the acetyl group $\left(\mathrm{CH}_{3} \mathrm{COO}^{-}\right)$of acetylCoA to the $\varepsilon$-amino group $\left(\mathrm{NH}_{3}\right)$ of specific lysine residues at the $\mathrm{N}$ terminus of core histones (mainly $\mathrm{H} 3$ and $\mathrm{H} 4)$. Histone acetylation can neutralize the positive charge on lysine residues, weaken the binding of histones to DNA, loosen the structure of chromatin, and facilitate the binding of transcription factors or transcriptional regulatory proteins to DNA, thereby promoting gene transcription [4-7]. In general, HAT-mediated histone acetylation is reported to be associated with gene upregulation, but this process has been little studied in plants and requires further research [8].

Histone acetylation is important for the modification of chromatin structure in eukaryotic cells, affecting gene transcription and thereby playing a crucial regulatory role in plant development. Plant HATs are classified into four families. HACs are similar to the p300/CREB (cAMP responsive element-binding protein)-binding protein (CBP) family. HAFs are related to the TATA-binding proteinassociated factor (TAFII250) family and HAMs to the MOZ, Ybf2/Sas3, Sas2, and Tip60 (MYST) family. Finally, HAGs are related to the general control non-repressible 5related N-terminal acetyltransferase (GNAT) family with an acetyltransf_1 (AT1) domain (PF00583) and include GCN5-, ELP3-, and HAT1-like acetyltransferases [9]. As yet, HATs have been identified in several model plant species, including Arabidopsis thaliana [3], Oryza sativa [10], barley [11], Vitis vinifera [12], tomato [13], litchi [14], and Zea mays [15]. Silencing of AtHAM1 and AtHAM2 in A. thaliana induces severe defects in the formation of male and female gametophytes [16]. It is essential for root stem cell niche maintenance that $A t G C N 5$ upregulates the expression of the root stem cell transcription factors PLET HORA1 (PLT1) and PLT2 [17]. Mutations in AtGCN5 and AtHAF2 lead to reduced expression of light-responsive genes $[18,19]$. Loss of function of AtHAC1, AtHAC5, and AtHAC12 causes delayed flowering phenotypes [20, 21]. These findings indicate that histone acetylation plays a crucial role in the control of plant development.
Plants encounter various environmental stimuli during their life cycle, including abiotic and biotic stresses. Plant response to various environmental stresses depends largely on posttranslational nucleosome histone modifications, including histone acetylation [22]. Histone acetylation participates in the temperature regulation of plant development, and cold exposure represses the expression of four HATs (OsHAC701, OsHAC703, OsHAC704, and OsHAG703) in $O$. sativa [10]. In $A$. thaliana, physical interaction of AtGCN5 with the cold-induced transcription factor CBF1 (a $\mathrm{C}$ repeat/DRE binding factor) through the transcriptional coactivator ADA2b (a homolog of yeast ADA2 protein) regulates the cold accumulation process of cold-regulated (COR) gene expression [23]. Histone acetylation is also involved in the response of plants to biotic stress. In Magnaporthe oryzae, growth rate and spore production are significantly reduced in MoHat1 knockout mutants, reducing their ability to infect plants [24]. A HAT gene (PsGcn5) from Phytophthora sojae is important for growth under conditions of oxidative stress and contributes to full virulence by suppressing host-derived reactive oxygen species [25]. SAGA (Spt-Ada-Gcn5-acetyltransferase) participates in the regulation of dicer-like2 (DCL2)-mediated transcriptional response, thereby regulating the RNAi pathway of Cryphonectria parasitica [26].

Although HATs have multiple roles in plant growth, development and stress response, little is known about their functions during viral infection, especially in wheat (Triticum aestivum). Wheat is the most widely grown crop around the globe and ranks second in importance to rice for food. However, in comparison with rice and maize, wheat is under-explored [27]. In this study, we identified and characterized members of the HAT gene family in $T$. aestivum and comprehensively analyzed their phylogenetic relationships, structures, chromosomal locations, expression patterns, responses to temperature stress, and responses to viral inoculation. Taken together, our results provide a set of TaHAT genes that have particular roles in the response to viral infection for future studies in plant immunity.

\section{Results \\ Identification and characterization of HAT genes in $T$. aestivum}

Previous studies have shown that there are 12 HATs in A. thaliana and eight HATs in O. sativa $[3,10]$. Here, we identified 31 HATs in wheat (T. aestivum) by performing BLASTP searches with $A$. thaliana and $O$. sativa HAT protein sequences as queries (Table S1). HATs belong to four distinct classes: HAC, HAG, HAF, and HAM [3]. According to their conserved domains and the classification of HATs in A. thaliana, the 31 TaHATs were divided into six classes for convenient description: HAC, HAG1, HAG2, HAG3, HAF, and HAM. 
Each class has distinct conserved domains that support the suitability of such a grouping (Fig. 1). Details of the TaHAT gene family, including gene IDs, locations, and groups are provided in Table 1. Most TaHACs (85\%) were 1100-1800 aa in length while TraesCS6B02G367300.1 (484 aa) and TraesCS6D02G317200.1 (607 aa) were special. The amino acid sequences of each class showed a high similarity. The molecular weights (MWs) of the TaHATs varied from 50.14 to $201.47 \mathrm{kDa}$. The isoelectric points (pIs) ranged from 5.23 to 8.88. TraesCS7A02G514800.1 encoded the longest protein with the highest MW (201.47), whereas TraesCS2A02G159700.1 and TraesCS2D02G166900.1 encoded the shortest proteins with the lowest MWs (50.14) (Table 1). The protein properties of the TaHATs were similar to those of HATs from other plant species [3, 10].

\section{Phylogenetic analysis of the HAT proteins}

To analyze the phylogenetic relationships among HATs from different species, $12 \mathrm{~A}$. thaliana (diploid), eight $O$. sativa (tetraploid), and $31 \mathrm{~T}$. aestivum (hexaploid) HAT protein sequences were used to construct a neighborjoining (NJ) tree. Unrooted trees that make no assumptions about ancestry illustrate only the relationships among the leaf nodes [28]. As shown in Fig. 2, HAT proteins from the three species were divided into six clades, as expected. The TaHAT proteins shared high homology with HAT proteins from other species. They clustered into the same clades with AtHATs and OsHATs with high bootstrap support values. One AtHAT, one OsHAT, and three TaHATs were clustered into groups HAG1, HAG2, and HAG3. Regardless of species, HAC was the largest group, with five AtHATs, three OsHATs, and 13 TaHATs. One AtHAT and one OsHAT were assigned to the HAM and HAF groups. The HAF group had three more TaHATs than the HAM group. These results are consistent with two previous studies of HATs from $A$. thaliana and O. sativa, which documented similar phylogenetic relationships among these proteins [3, 10, 28].

\section{Predicted structure analysis of HAT proteins}

Homology modelling has matured into an important technique in structural biology [29]. To visualize the various structures, we selected a random protein from each group in three species and modeling by SWISSMODEL. HAC, HAG2, and HAG3 proteins had similar structures in different species. HAM, HAF, and HAG1 protein structures seemed to differ among species, but a closer look revealed that the conserved protein structures were complete and only the folding directions differed slightly (Fig. 3). As TaHACs contain two special genes which miss partial introns, we also performed protein modeling for them by SWISS-MODEL. The result showed that these two genes have similar protein structures to other family members of TaHAC (Figure S1). In general, the models of proteins from the same groups in different species were very similar, whereas those of proteins from different groups within the same species were different.

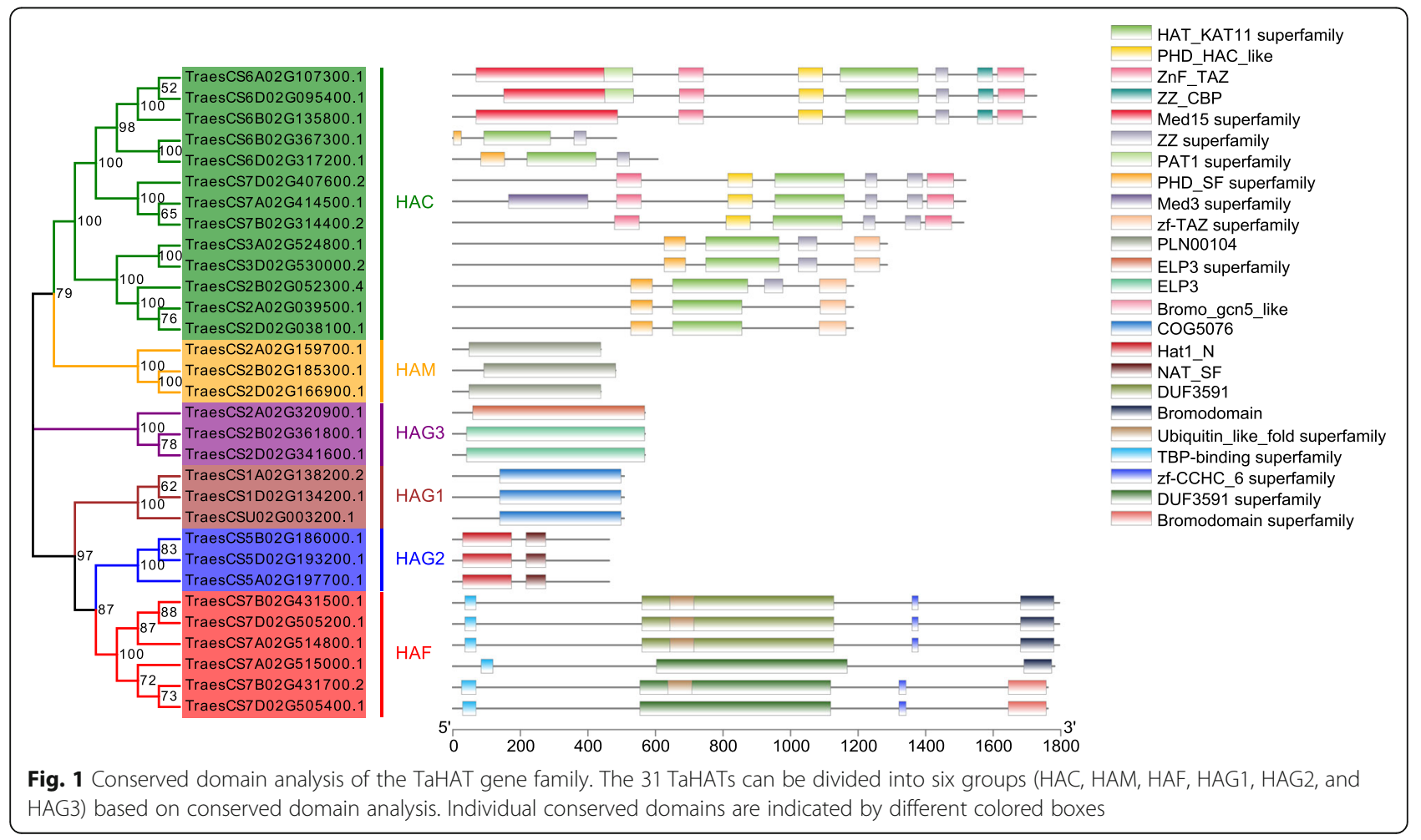


Table 1 Detailed information about 31 predicted HATs proteins in Triticum aestivum

\begin{tabular}{|c|c|c|c|c|c|c|c|}
\hline Gene ID & Location & CDS Length (bp) & Size (aa) & MW (kDa) & PI & Exons & Groups \\
\hline TraesCS2A02G039500.1 & $2 A: 16464148-16,472,158$ & 3558 & 1185 & 133.28 & 6.82 & 17 & $H A C$ \\
\hline TraesCS2B02G052300.4 & $2 B: 25594256-25,602,539$ & 3561 & 1186 & 133.64 & 7 & 17 & $H A C$ \\
\hline TraesCS2D02G038100.1 & 2D:14091001-14,099,684 & 3558 & 1185 & 133.24 & 6.7 & 17 & $H A C$ \\
\hline TraesCS3A02G524800.1 & 3A:739388676-739,397,596 & 3861 & 1286 & 144.36 & 7.54 & 17 & $H A C$ \\
\hline TraesCS3D02G530000.2 & 3D:606995302-607,004,223 & 3861 & 1286 & 144.61 & 7.53 & 17 & $H A C$ \\
\hline TraesCS6A02G107300.1 & $6 A: 75905141-75,917,538$ & 5181 & 1726 & 194.15 & 8.62 & 16 & $H A C$ \\
\hline TraesCS6B02G135800.1 & 6B:1331 16840-133,127,600 & 5181 & 1726 & 194.20 & 8.6 & 17 & $H A C$ \\
\hline TraesCS6B02G367300.1 & 6B:641295622-641,299,743 & 1455 & 484 & 55.69 & 6.36 & 9 & $H A C$ \\
\hline TraesCS6D02G095400.1 & 6D:59617005-59,629,170 & 5187 & 1728 & 194.26 & 8.64 & 16 & $H A C$ \\
\hline TraesCS6D02G317200.1 & 6D:426068136-426,073,461 & 1824 & 607 & 70.10 & 6.16 & 10 & $H A C$ \\
\hline TraesCS7A02G414500.1 & 7A:605759167-605,772,416 & 4557 & 1518 & 172.36 & 8.82 & 18 & $H A C$ \\
\hline TraesCS7B02G314400.2 & 7B:561735305-561,748,672 & 4539 & 1512 & 171.76 & 8.88 & 18 & $H A C$ \\
\hline TraesCS7D02G407600.2 & 7D:525362988-525,375,228 & 4557 & 1518 & 172.31 & 8.9 & 17 & $H A C$ \\
\hline TraesCS7A02G514800.1 & 7A:700689132-700,707,173 & 5391 & 1796 & 201.47 & 5.36 & 21 & $H A F$ \\
\hline TraesCS7A02G515000.1 & 7A:700795547-700,810,249 & 5349 & 1782 & 199.72 & 5.43 & 20 & HAF \\
\hline TraesCS7B02G431500.1 & 7B:699821564-699,839,075 & 5391 & 1796 & 201.46 & 5.38 & 21 & HAF \\
\hline TraesCS7B02G431700.2 & 7B:699909753-699,924,144 & 5289 & 1762 & 198.37 & 5.32 & 20 & HAF \\
\hline TraesCS7D02G505200.1 & 7D:610806229-610,820,455 & 5391 & 1796 & 201.44 & 5.35 & 21 & HAF \\
\hline TraesCS7D02G505400.1 & 7D:610841068-610,855,207 & 5289 & 1762 & 197.56 & 5.23 & 20 & HAF \\
\hline TraesCS5D02G193200.1 & 5D:297497242-297,502,158 & 1392 & 463 & 51.43 & 4.71 & 10 & HAG2 \\
\hline TraesCS5B02G186000.1 & 5B:337828291-337,833,473 & 1392 & 463 & 51.41 & 4.79 & 11 & HAG2 \\
\hline TraesCS5A02G197700.1 & 5A:401745642-401,754,640 & 1392 & 463 & 51.60 & 4.88 & 10 & HAG2 \\
\hline TraesCS2A02G320900.1 & $2 A: 550539215-550,542,666$ & 1710 & 569 & 63.57 & 8.88 & 9 & HAG3 \\
\hline TraesCS2B02G361800.1 & 2B:514861001-514,865,480 & 1710 & 569 & 63.61 & 8.88 & 10 & HAG3 \\
\hline TraesCS2D02G341600.1 & 2D:436369113-436,372,717 & 1710 & 569 & 63.58 & 8.88 & 9 & HAG3 \\
\hline TraesCS1A02G138200.2 & $1 \mathrm{~A}: 230132869-230,165,911$ & 1524 & 507 & 56.49 & 6.34 & 13 & HAG1 \\
\hline TraesCS1D02G134200.1 & 1D:166822476-166,847,564 & 1524 & 507 & 56.47 & 6.25 & 13 & HAG1 \\
\hline TraesCSU02G003200.1 & Un:5332971-5,357,477 & 1524 & 507 & 56.51 & 6.34 & 13 & HAG1 \\
\hline TraesCS2A02G159700.1 & $2 A: 107382526-107,388,480$ & 1317 & 438 & 50.14 & 7.21 & 9 & HAM \\
\hline TraesCS2B02G185300.1 & 2B:160401169-160,407,504 & 1449 & 482 & 54.94 & 6.77 & 9 & HAM \\
\hline TraesCS2D02G166900.1 & 2D:1 10937430-110,943,241 & 1317 & 438 & 50.14 & 7.21 & 9 & HAM \\
\hline
\end{tabular}

CDS coding sequence, $b p$ base pair, $a a$ amino acids, $M W$ molecular weight, $D a$ Dalton, $P I$ isoelectric point

\section{Structures and conserved motifs of the TaHATs}

Since the comparison of gene structures provides insight into gene family evolution, we analyzed the structures of the TaHAT genes [30]. Analysis of gDNA sequences showed that the number of introns ranged from 2 to 14 (Fig. 4). The TaHATs with highly similar gene structures were clustered together in the six main branches of the NJ tree. Most TaHATs had similar numbers of introns and exons, with the exception of two TaHAC genes (TraesCS6B02G367300.1 and TraesCS6D02G317200.1). The TaHAFs had the highest number of introns (14) among all the groups.

To characterize putative motifs in the wheat HAT family, the predicted amino acid sequences of the 31
TaHAT proteins were submitted to the MEME website. The result showed that 20 conserved motifs were predicted in these proteins (Fig. 5). Members of the same group contained similar motifs, suggesting that these proteins may have similar functions [31]. The HAC group had the largest number of motifs. There were probably 10 motifs in each protein, and they were arranged in the same order in the majority of sequences (motif 6 , motif 10 , motif 2 , motif 1 , motif 9 , and motif 5). Motifs $1,3,7,8,11$, and 20 were present only in the HAC group. Other groups also had their own unique motif sequences, and details of the 20 conserved motifs are presented in Table S2. 


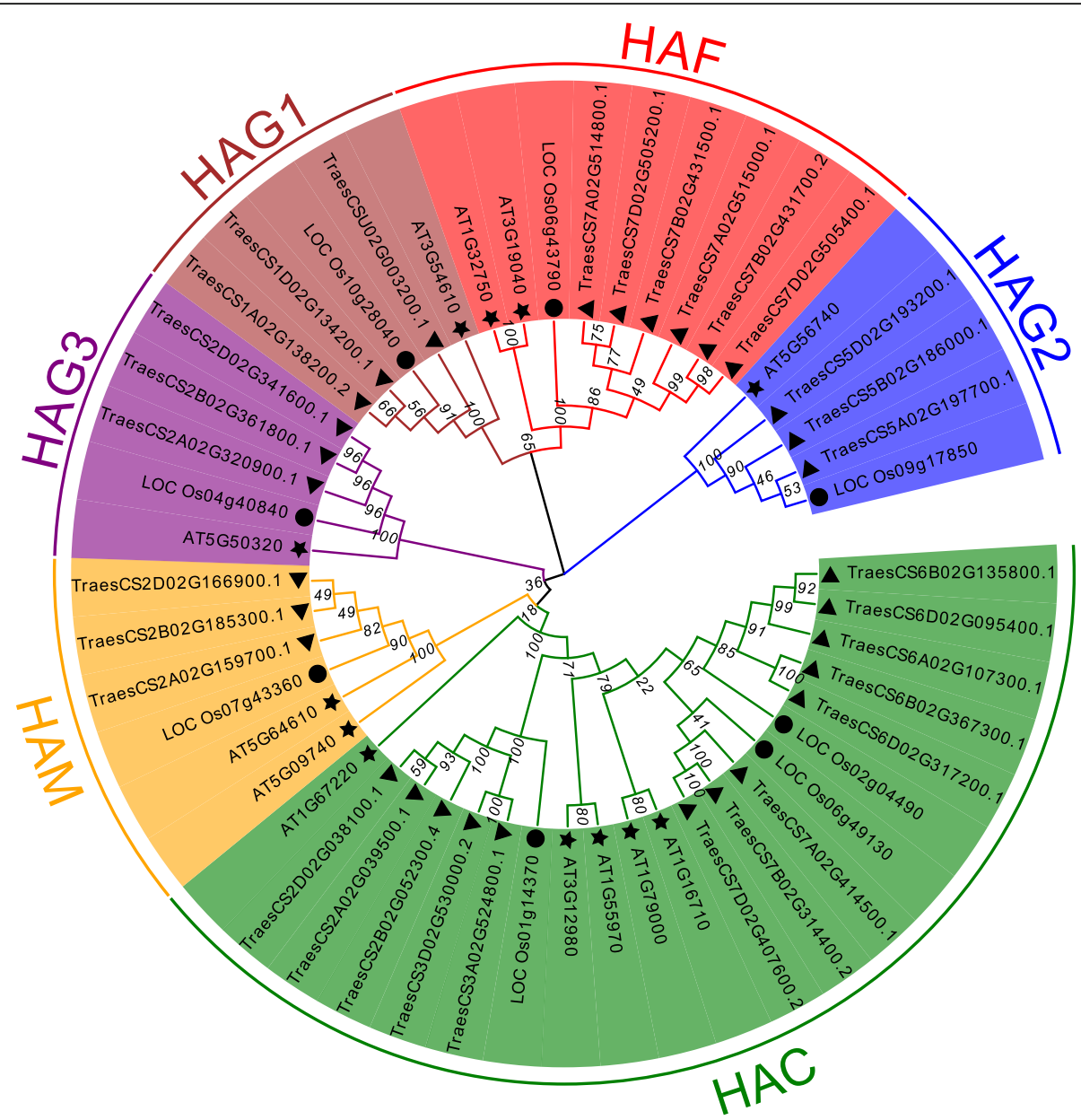

Fig. 2 Phylogenetic tree of HAT proteins from Arabidopsis thaliana, Oryza sativa and Triticum aestivum constructed by the neighbor-joining method in MEGA-X. The numbers at nodes represent bootstrap values after 1000 iterations. Each group is indicated by a different color. Stars represent $A$. thaliana, circles represent $O$. sativa, and triangles represent $T$. aestivum

\section{Chromosomal locations and Synteny analysis of the TaHATs}

The TaHAT genes were distributed unevenly among the chromosomes of the T. aestivum genome (Fig. 6 and Figure S2). Three TaHATs were distributed on chromosomes 2A, 2B, 2D, 7A, 7B, and 7D. Two TaHATs were distributed on chromosome $6 \mathrm{~B}$ and $6 \mathrm{D}$ while no TaHAT gene was found on chromosome 3B, 4A, 4B, 4D. Tandem and segmental gene duplications are commonly found in plant genomes [32]. Based on synteny analysis and the inspection of gene duplications, the 31 TaHATs can be summarized as 12 genes (five $H A C \mathrm{~s}$, two $H A F \mathrm{~s}$, two HAG1s, one HAM, one HAG2 and one HAG3), including eight genes with three copies, three genes with two copies and one gene with one copy.

\section{Calculation of TaHAT duplication events}

In genetics, $\mathrm{Ka} / \mathrm{Ks}$ represent the ratio between the nonsynonymous substitution rate $(\mathrm{Ka})$ and the synonymous substitution rate (Ks) of two protein-coding genes. This ratio can determine whether there is selective pressure acting on the gene [33]. Collinearity and synteny analyses of chromosomes identified 21 putative paralogs in wheat $(\mathrm{Ta}-\mathrm{Ta})$ (Table 2) and 20 putative orthologs between wheat and rice $(\mathrm{Ta}-\mathrm{Os})$ (Table 3). All $\mathrm{Ta}-\mathrm{Ta}$ pairs were located on homologous chromosomes (Chr2, Chr5, Chr6 and Chr7). $H A T$ pairs were considered to be under purifying selection when $\mathrm{Ka} / \mathrm{Ks}$ of either paralogs or orthologs were less than one. A mutation that changes a protein is less likely to differ between two species than one which is silent. Most of the time, plant eliminates deleterious mutations to avoid the protein mutation [33]. The divergence time ( $\mathrm{T})$ was assessed as $\mathrm{T}=\mathrm{Ks} /$ $\left(2 \times 9.1 \times 10^{-9}\right)$ million years age (Mya) based on a divergence rate of $9.1 \times 10^{-9}$ synonymous mutations per synonymous locus per year [33]. The 21 paralogous pairs $(T a-T a)$ were assessed to have diverged between 0.845 and 4.385 Mya and the 20 orthologous pairs ( $\mathrm{Ta}-\mathrm{Os}$ ) between 23.146 and 62.318 Mya. 


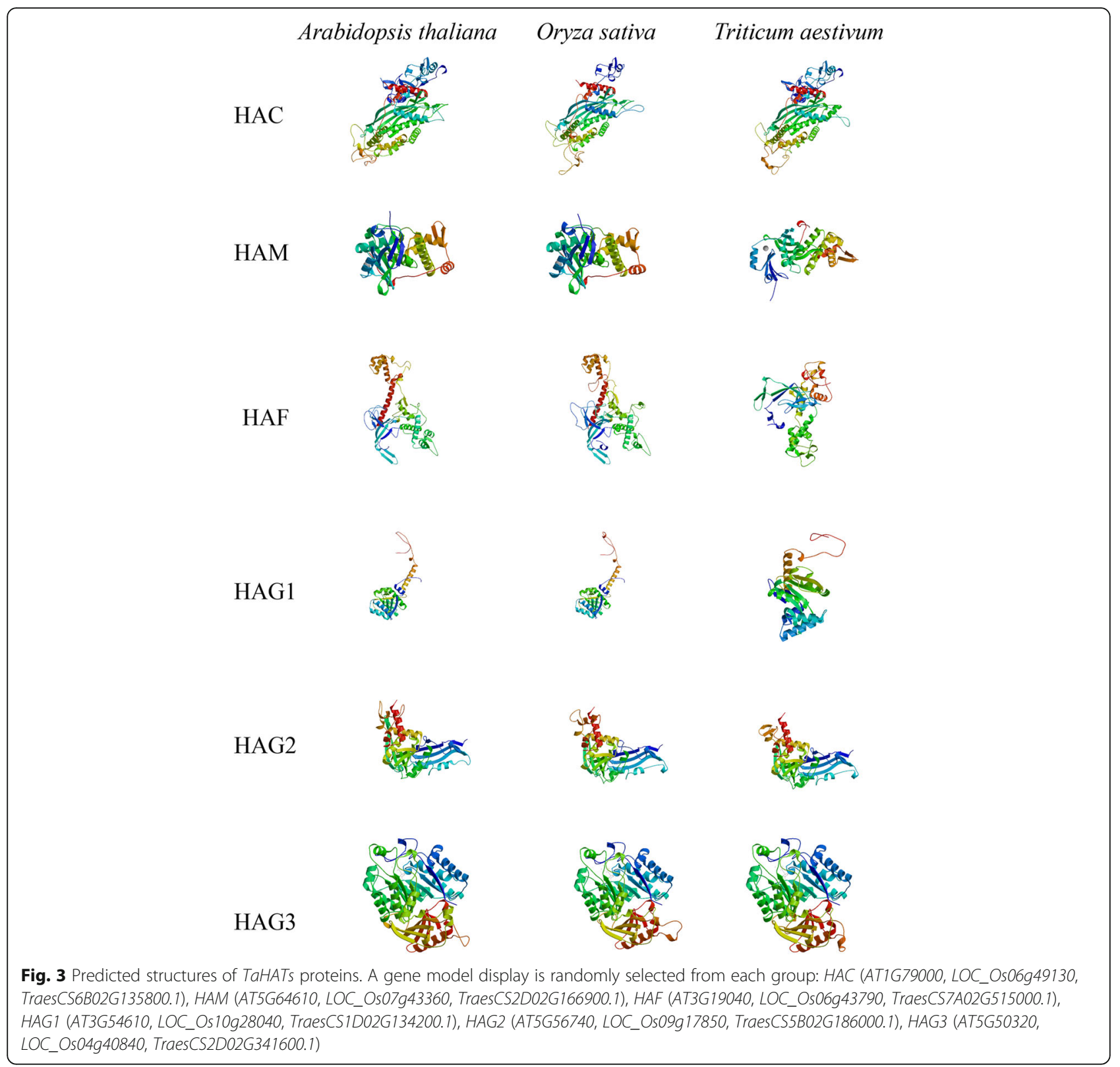

\section{Expression profiles of TaHATs in three-leaf-stage wheat}

$H A T$ s play a role in plant development [8]. To study the expression patterns of the TaHAT genes, one TaHAT from each subfamily was chosen at random for expression analysis in three-leaf-stage seedlings using quantitative real-time PCR (qRT-PCR). The plants were divided into five tissue types: top leaf, middle leaf, bottom leaf, stem, and roots. As shown in Fig. 7 and Figure S3, six TaHAT genes were expressed in different tissues. In addition to HAG1, other genes also showed high expression levels in roots. All genes showed moderate expression levels in the stem. Interestingly, gene expression levels in the bottom leaf and the top leaf were higher than those in the middle leaf. In general, faster growing wheat tissues had a higher relative expression level of $H A T$ genes. These results indicate that the expression pattern of TaHATs differs among tissues and is related to plant development.

\section{Prediction and analysis of cis-acting elements in the promoter regions of TaHAT genes}

A total of 1643 cis-acting elements were predicted in the promoter regions of the TaHAT genes. These elements associated with environmental stress, hormone response, light response, development, promoter and enhancer elements, site-binding elements, and others (Fig. 8). Hormone responsive elements were the most abundant, including auxin (IAA), gibberellin (GA), salicylic acid 


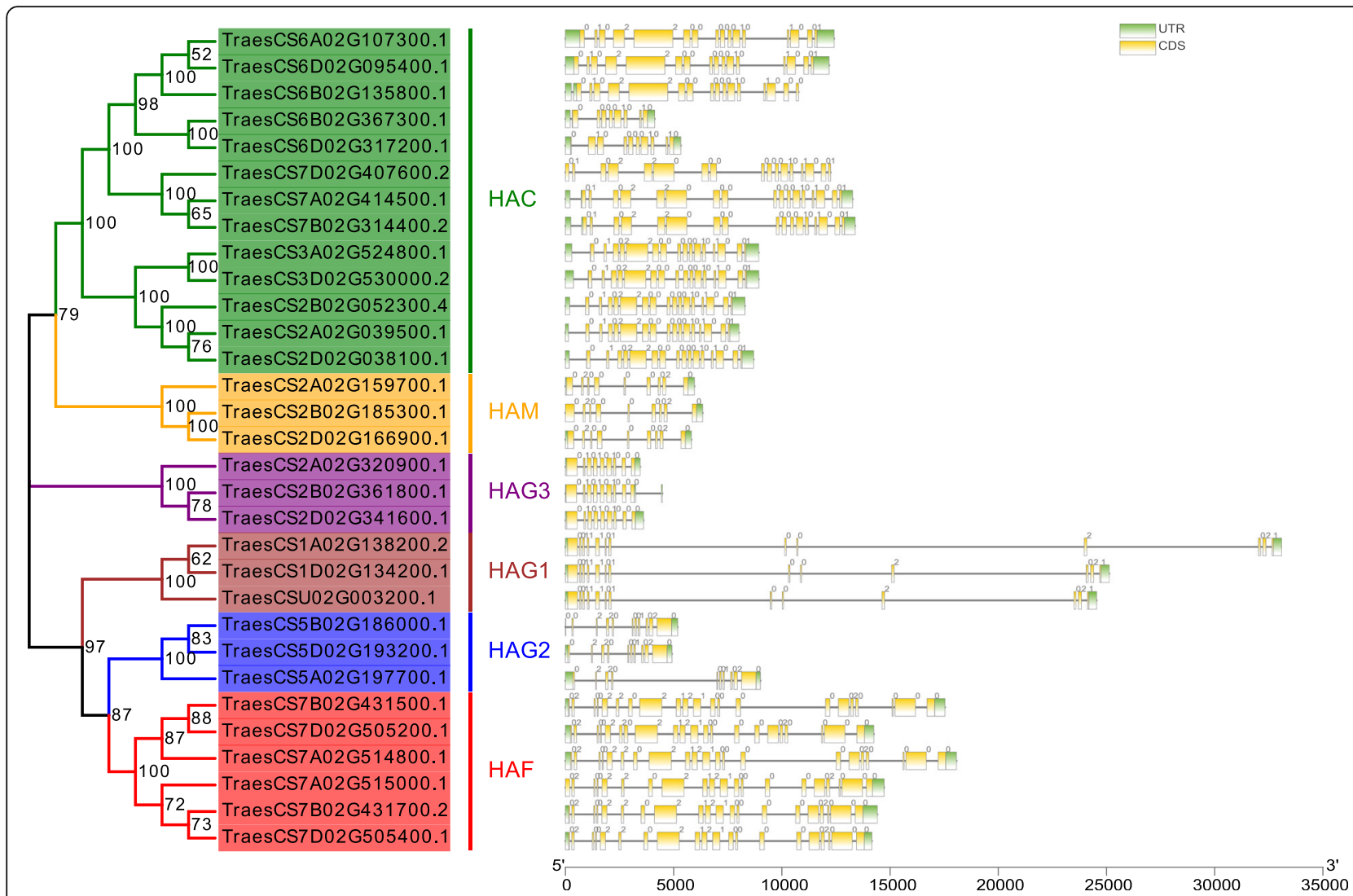

Fig. 4 Exon-intron structures of 31 TaHAT genes. Exons, introns, and untranslated regions (UTR) are indicated by yellow frames, gray lines, and green frames on the right, respectively. The number on the gray line represents the number of introns

(SA), abscisic acid (ABA) and methyl jasmonate (MeJA). Plant growth and development are affected by various environmental stresses. Therefore, it is important to study the cis-acting elements associated with environmental stress [34]. In this study, 88 elements were related to environmental stress, including 25 lowtemperature-responsive elements, 10 defense- and stress-responsive elements and 53 elements essential for anaerobic induction.

\section{Expression patterns of TaHATs under different stresses}

Studies have shown that the expression levels of HAT genes are affected by plant hormones, low temperature, drought, and salt stress [9]. To confirm that the expression of TaHAT genes could be regulated by abiotic and biotic stress, we tested the effects of low temperature as an abiotic stress and virus inoculation as biotic stress. The expression patterns of six TaHAT genes in the second leaves of 10-14 day old wheat were measured by qRT-PCR.

The relative expression levels of TaHAT genes were different when wheat developed at different temperatures. Most TaHAT genes showed low expression at low temperatures over 7 to 10 days treatment. Among them,
TaHAC, TaHAF and TaHAG1 showed significantly lower expression level at $8{ }^{\circ} \mathrm{C}$ compared with other temperatures. As treatment time increased, there were no significant differences in the relative expression of TaHAC, TaHAM and TaHAG3. However, the expression level of TaHAF, TaHAG1 and TaHAG2 were still lower at $8{ }^{\circ} \mathrm{C}$ than at $20^{\circ} \mathrm{C}$ (Fig. 9). In addition, TaHAT expression levels were upregulated at 16 days post infection (dpi) in wheat inoculated with barley streak mosaic virus (BSMV), Chinese wheat mosaic virus (CWMV), or wheat yellow mosaic virus (WYMV). The expression levels of most TaHATs increased from 7 to $16 \mathrm{dpi}$ (Fig. 10). Reverse transcription PCR (RT-PCR) detects whether the three viruses successfully infect wheat (Figure S4).

\section{Discussion}

Members of the HAT family usually exist in the form of complexes and play a very important regulatory role in multiple cellular processes, including transcriptional activation, gene silencing, cell cycle regulation, DNA replication and repair, and chromosome assembly [35, 36]. HAT activity is closely related to plant growth, development, stress response, and the cell cycle process $[37,38]$. 

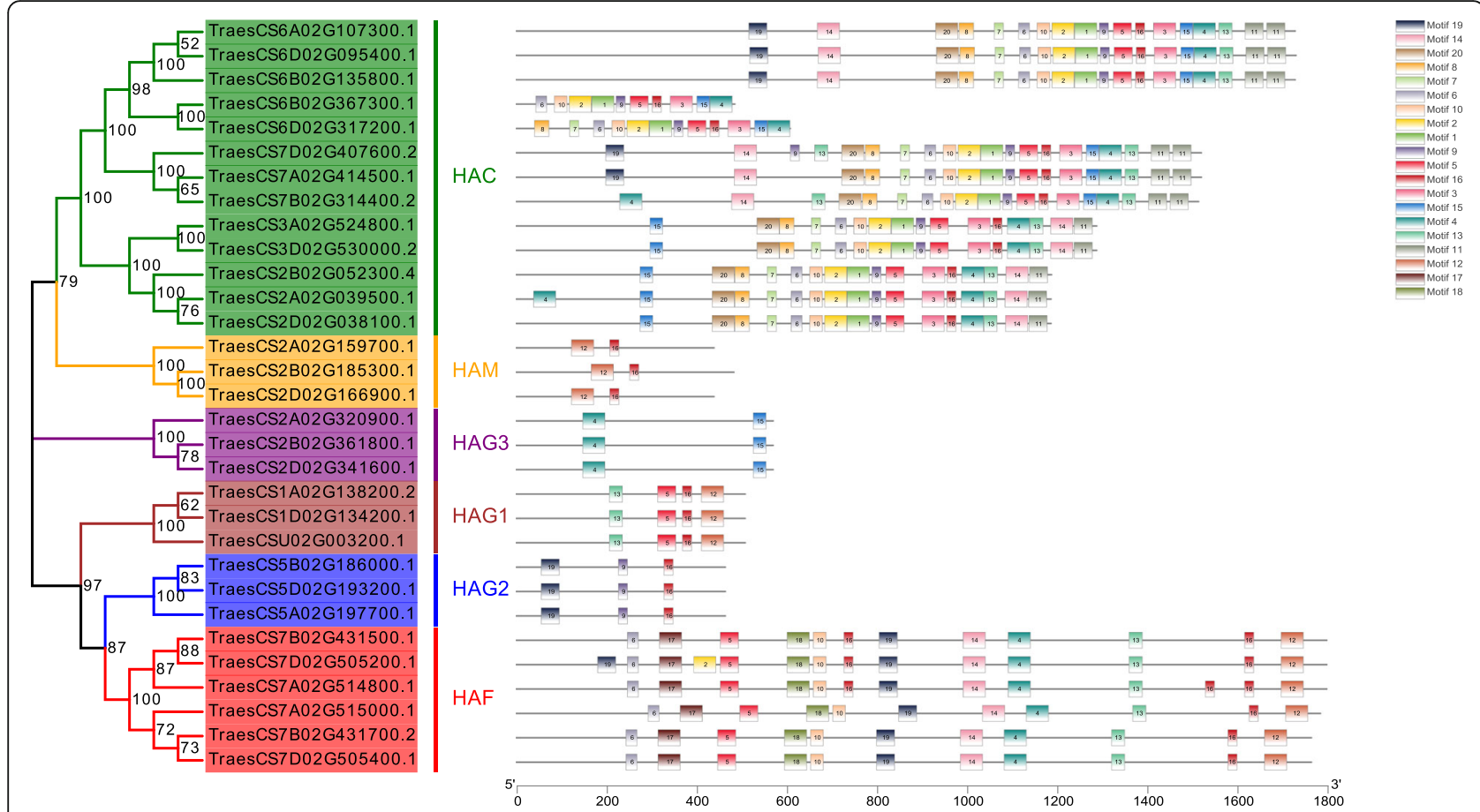

Fig. 5 Schematic representation of twenty conserved motifs in the TaHATs. Different colored frames represent different protein motifs, and each motif has its own number

HAT gene families have been identified and analyzed in several plants, including A. thaliana [3], O. sativa [10], barley [11], $V$. vinifera [12], tomato [13], litchi [14] and $Z$. mays [15]. In this work, we identified $31 H A T \mathrm{~s}$ in $T$. aestivum and found that their structural domains were conserved by studying phylogenetic trees, gene structures and conserved motifs. The HAT genes could be divided into four families: HAC, HAF, HAM and HAG [3]. We further divided the HAGs into HAG1, HAG2 and HAG3 based on differences in their conserved domains. After predicting their protein structures, we found that the structures of HAG1, HAG2 and HAG3 were quite different. These results indicated that they may have different functions and supporting the proposed subgroups (Figs. 1, 2, 3, 4 and 5). TaHATs in each subgroup had similar gene structures and motifs, with the exceptions of the TaHACs TraesCS6B02G367300.1 and TraesCS6D02G317200.1 (Figs. 4 and 5). However, the major domains and structures of their encoded proteins were the same as those of other TaHACs (Fig. 1). We speculated that these two genes lost introns through duplication events over evolutionary time, as this is a common phenomenon [28]. On the basis of chromosome locations, we found that most genes were present in three copies, consistent with the existence of three homologous chromosomes in the wheat genome. Genes that have fewer than three copies may have experienced gene loss over the course of evolution (Fig. 6). During the evolution of TaHATs, duplicated genes may have lost functions or gained new functions [39]. Notably, 31 $H A T$ s were identified in the wheat genome, whereas only 12 and 8 are present in A. thaliana and O. sativa, respectively $[3,10]$. This result indicates that a higher level of HAT gene duplication occurred during the evolution of wheat and may be attributed to wheat's allohexaploid genome and complex evolution [40].

Although histone modifications are thought to play an important role in growth, development processes and stress responses, the specific functions of HATs in T. aestivum remain unknown [9]. Many previous studies have shown that ortholog analysis is a feasible method for predicting the unknown functions of homologous genes from different species [41]. Since orthologs are derived from a single gene in the last common ancestor of two or more species, they often have the same function in newly evolved taxa [40]. Therefore, to predict the potential biological roles of the TaHATs, we reviewed known examples of these enzymes in A. thaliana and $O$. sativa to perform functional identification, and we determined the closest wheat ortholog based on phylogenetic analysis (Fig. 2). We also built protein models for each group and found that protein structures were similar within groups, demonstrating the suitability of this method for comparisons of different species (Fig. 3). In vivo, AtHAC1, AtHAC5 and AtHAC12 play redundant roles in the promotion of flowering by inhibiting the 


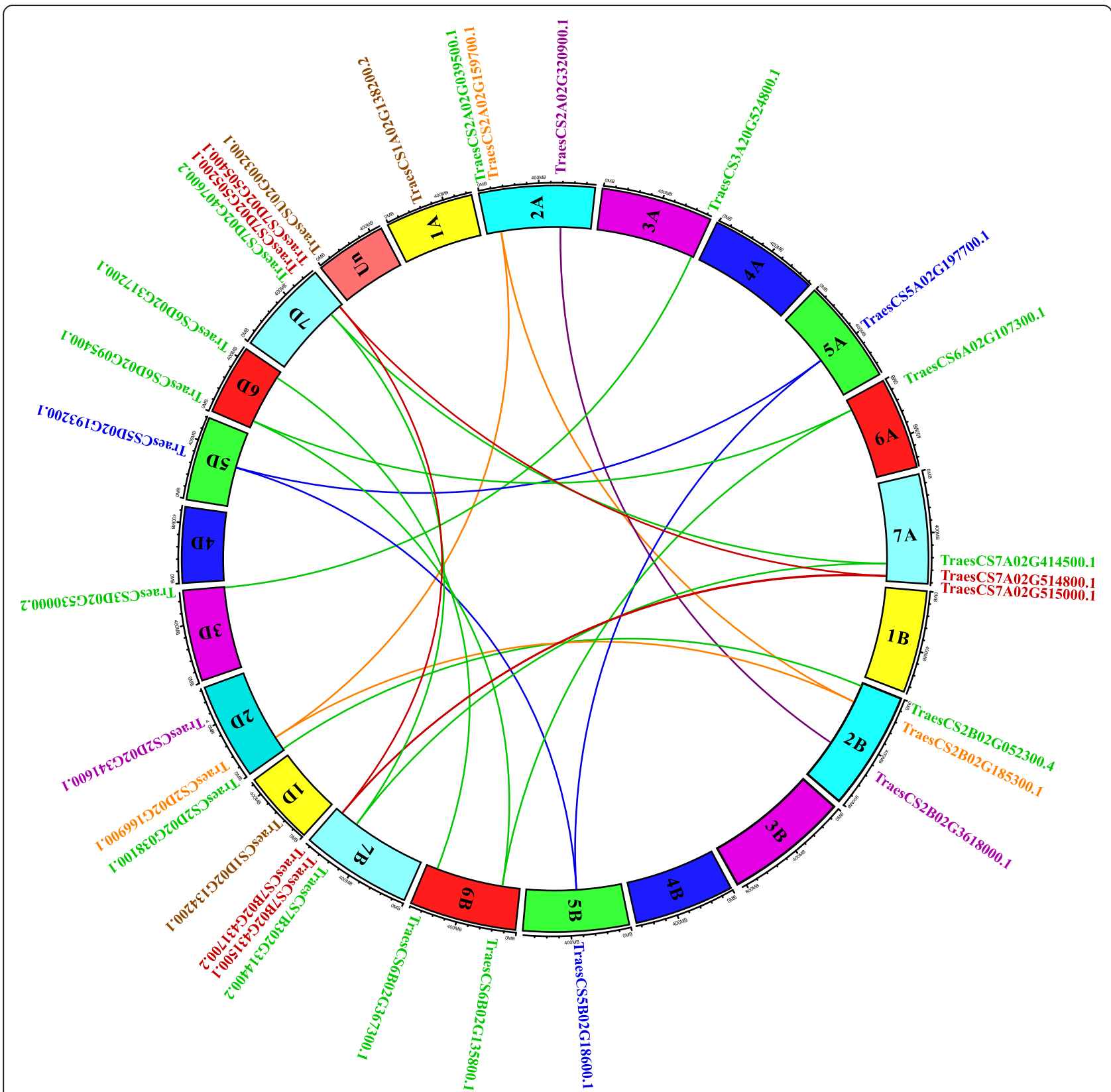

Fig. 6 Chromosomal Locations and Synteny Analysis of TaHATs. Homologous chromosomes are filled with the same color. The different colored lines indicate duplicated TaHAT gene pairs on different chromosomes. The positions of TaHATs are marked directly on the chromosomes, and the different colored fonts represent different groups: HAC (green), HAM (orange), HAF (red), HAG1 (brown), HAG2 (blue), and HAG3 (purple)

expression of FLC (FLOWERING LOCUS C) [20, 21]. Similarly, it has been reported that AtHAC1 regulates factors upstream of FLC at flowering time through epigenetic modification and also interacts with the tomato heat stress transcription factor $\mathrm{HsfB} 1$ in vitro and in vivo [42]. The TaHAC homologs with AtHACs may therefore influence flowering in wheat (Figs. 2 and 3). Since AtHAM1 and AtHAM2 are involved in the formation of male and female gametophytes [43], we hypothesize that the five closest TaHAM orthologs may perform the same function (Figs. 2 and 3). RNAimediated AtHAF1 gene silencing in A. thaliana confers resistance to Agrobacterium-mediated transformation [44]. AtHAF2 regulates the expression of several coldregulated genes, regardless of its HAT activity [45]. Based on phylogenetic analysis and similarities in their protein models, TaHAFs may have similar functions to AtHAF1 and AtHAF2 (Figs. 2 and 3). AtHAG1 plays a crucial role in cell differentiation and leaf and flower organ formation [46]. TaHAG1 is highly expressed in 
Table $2 \mathrm{Ks}, \mathrm{Ka}$, and Ka/Ks values calculated for paralogous HAT gene-pairs (T. aestivum - T. aestivum)

\begin{tabular}{|c|c|c|c|c|c|}
\hline Paralogous pairs & & $\mathrm{Ka}$ & Ks & $\mathrm{Ka} / \mathrm{Ks}$ & T (Mya) \\
\hline TraesCS2B02G052300.4 & TraesCS2D02G038100.1 & 0.0113 & 0.0452 & 0.2505 & 2.485 \\
\hline TraesCS3A02G524800.1 & TraesCS3D02G530000.2 & 0.0108 & 0.0317 & 0.3399 & 1.743 \\
\hline TraesCS6A02G107300.1 & TraesCS6B02G135800.1 & 0.0027 & 0.0277 & 0.0990 & 1.519 \\
\hline TraesCS6A02G107300.1 & TraesCS6D02G095400.1 & 0.0040 & 0.0249 & 0.1601 & 1.365 \\
\hline TraesCS6B02G135800.1 & TraesCS6D02G095400.1 & 0.0037 & 0.024 & 0.1555 & 1.320 \\
\hline TraesCS6B02G367300.1 & TraesCS6D02G317200.1 & 0.0173 & 0.0445 & 0.3877 & 2.447 \\
\hline TraesCS7A02G414500.1 & TraesCS7B02G314400.2 & 0.0149 & 0.0529 & 0.2812 & 2.906 \\
\hline TraesCS7A02G414500.1 & TraesCS7D02G407600.2 & 0.0059 & 0.0368 & 0.1612 & 2.023 \\
\hline TraesCS7B02G314400.2 & TraesCS7D02G407600.2 & 0.0146 & 0.0486 & 0.3004 & 2.668 \\
\hline TraesCS7A02G514800.1 & TraesCS7D02G505200.1 & 0.0063 & 0.0587 & 0.1081 & 3.227 \\
\hline TraesCS7A02G514800.1 & TraesCS7B02G431500.1 & 0.0069 & 0.0610 & 0.1140 & 3.349 \\
\hline TraesCS7A02G515000.1 & TraesCS7B02G431700.2 & 0.0248 & 0.0413 & 0.6014 & 2.270 \\
\hline TraesCS7B02G431500.1 & TraesCS7D02G505200.1 & 0.0092 & 0.0516 & 0.1783 & 2.836 \\
\hline TraesCS5B02G186000.1 & TraesCS5D02G193200.1 & 0.0047 & 0.0154 & 0.3076 & 0.845 \\
\hline TraesCS5A02G197700.1 & TraesCS5B02G186000.1 & 0.0143 & 0.0507 & 0.2814 & 2.783 \\
\hline TraesCS5A02G197700.1 & TraesCS5D02G193200.1 & 0.0133 & 0.0606 & 0.2195 & 3.328 \\
\hline TraesCS2A02G320900.1 & TraesCS2B02G361800.1 & 0.0016 & 0.0798 & 0.0197 & 4.385 \\
\hline TraesCS2A02G159700.1 & TraesCS2B02G185300.1 & 0 & 0.0691 & 0 & 3.796 \\
\hline TraesCS2A02G159700.1 & TraesCS2D02G166900.1 & 0 & 0.0372 & 0 & 2.042 \\
\hline TraesCS2B02G185300.1 & TraesCS2D02G166900.1 & 0 & 0.0617 & 0 & 3.393 \\
\hline
\end{tabular}

Table 3 Ks, Ka, and Ka/Ks values calculated for paralogous HAT gene-pairs (T. aestivum - Oryza sativa)

\begin{tabular}{|c|c|c|c|c|c|}
\hline Orthologs pairs & & Ка & Ks & $\mathrm{Ka} / \mathrm{Ks}$ & T (Mya) \\
\hline TraesCS7A02G414500.1 & LOC_Os02g04490.1 & 0.2824 & 1.1342 & 0.2490 & 62.318 \\
\hline TraesCS7A02G414500.1 & LOC_Os06g49130.1 & 0.2805 & 0.6306 & 0.4447 & 34.647 \\
\hline TraesCS7A02G514800.1 & LOC_Os06g43790.1 & 0.1095 & 0.5527 & 0.1982 & 30.370 \\
\hline TraesCS7B02G314400.2 & LOC_Os02g04490.1 & 0.2473 & 1.0127 & 0.2443 & 55.641 \\
\hline TraesCS7B02G314400.2 & LOC_Os06g49130.1 & 0.2822 & 0.5898 & 0.4786 & 32.404 \\
\hline TraesCS7B02G431500.1 & LOC_Os06g43790.1 & 0.1127 & 0.5477 & 0.2058 & 30.091 \\
\hline TraesCS6A02G107300.1 & LOC_Os02g04490.1 & 0.0871 & 0.4889 & 0.1781 & 26.863 \\
\hline TraesCS6A02G107300.1 & LOC_Os06g49130.1 & 0.2954 & 0.9333 & 0.3165 & 51.283 \\
\hline TraesCS6B02G135800.1 & LOC_Os02g04490.1 & 0.0874 & 0.488 & 0.1791 & 26.812 \\
\hline TraesCS6B02G135800.1 & LOC_Os06g49130.1 & 0.2942 & 0.9286 & 0.3168 & 51.023 \\
\hline TraesCS7D02G407600.2 & LOC_Os02g04490.1 & 0.2737 & 1.0781 & 0.2537 & 59.239 \\
\hline TraesCS7D02G407600.2 & LOC_Os06g49130.1 & 0.2874 & 0.6072 & 0.4734 & 33.362 \\
\hline TraesCS7D02G505200.1 & LOC_Os06g43790.1 & 0.1108 & 0.5354 & 0.2070 & 29.419 \\
\hline TraesCS6D02G095400.1 & LOC_Os02g04490.1 & 0.0880 & 0.4937 & 0.1782 & 27.128 \\
\hline TraesCS6D02G095400.1 & LOC_Os06g49130.1 & 0.2948 & 0.9341 & 0.3150 & 51.324 \\
\hline TraesCS2A02G320900.1 & LOC_Os04g40840.1 & 0.0171 & 0.4575 & 0.0374 & 25.138 \\
\hline TraesCS2A02G159700.1 & LOC_Os07g43360.1 & 0.0226 & 0.4213 & 0.0536 & 23.146 \\
\hline TraesCS2B02G361800.1 & LOC_Os04g40840.1 & 0.0171 & 0.4602 & 0.0372 & 25.284 \\
\hline TraesCS2B02G185300.1 & LOC_Os07g43360.1 & 0.0226 & 0.4746 & 0.0476 & 26.077 \\
\hline TraesCS2D02G166900.1 & LOC_Os07g43360.1 & 0.0226 & 0.4435 & 0.0560 & 24.367 \\
\hline
\end{tabular}




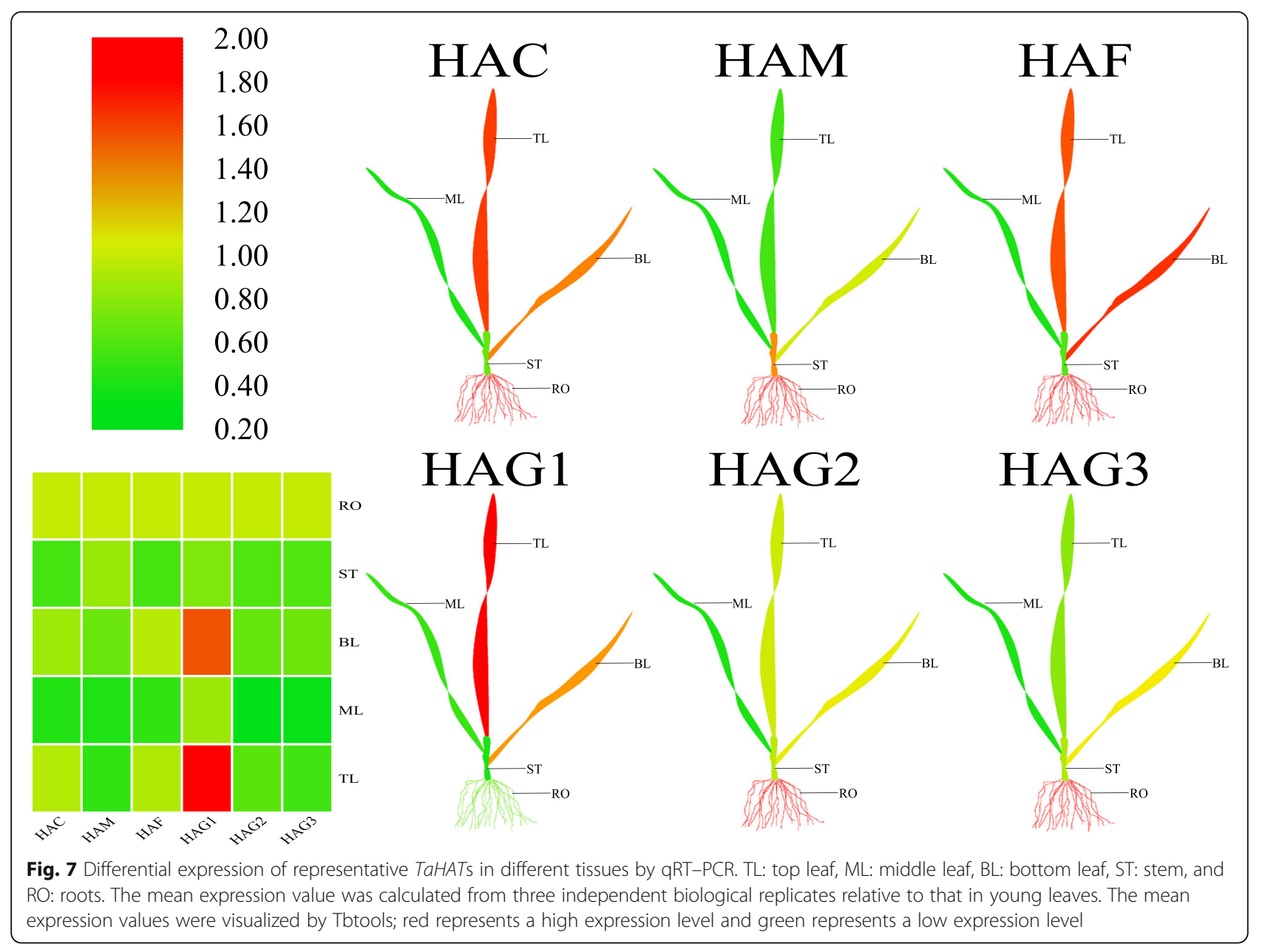

young leaves (Fig. 7) and may share similar function with AtHAG1 according to phylogenetic analysis and protein models results (Figs. 2 and 3). These results suggest that TaHAG1 may have a role in plant development. Histone H4K12 is acetylated by AtHAG2 [47], accordingly, the AtHAG2 homolog TaHAG2 may also perform the same function (Figs. 2 and 3). AtHAG3 interacts with RNA Pol II in the process of transcript extension and cell proliferation during organ growth [43]. AtHAG3 can also regulate plant response to ABA [48]. Moreover, AtHAG3 RNAi lines are resistant to Agrobacterium-mediated transformation [10, 44]. TaHAG3 may also have similar functions, as it clusters together with AtHAG3 in the phylogenetic tree and has a highly similar threedimensional structure (Figs. 2 and 3). These analyses strongly suggest that TaHATs play important roles in the growth and development of wheat. More detailed characterization of their functions can provide guidance for the cultivation of superior wheat varieties and for increasing wheat yield.

The relative expression levels of HATs change significantly under various biotic and abiotic stresses [9]. For example, in O. sativa, cold exposure represses the expression of four HATs (OsHAC701, OsHAC703, OsHAC704, and OsHAG703) [10]. The same phenomenon was observed here for all TaHAT genes (Fig. 9). In particular, TaHAF expression was upregulated 50- to 100-fold at 7 days of cold exposure. These results indicate that the expression level of TaHATs may be inhibited at low temperatures, thereby reducing the level of histone acetylation. However, as the treatment duration increased, the relative expression levels of TaHAC, TaHAM, and TaHAG3 at different temperatures ceased to be significant (Fig. 9). HAG1, HAG2 and HAG3 were reported to have different functions in Arabidopsis [49]. HAG2 is regulated by E2F transcription factors that induce the transcription of genes required for cell cycle progression and DNA replication [50]. Here, we found HAG2 sharply increased at $20^{\circ} \mathrm{C}$ at $16 \mathrm{~d}$ compared to those of other members. So we putative that HAG2 may participate in the regulation of some wheat genes expression at $20^{\circ} \mathrm{C}$. We speculate that these changes may reflect the acclimation of wheat to the environment. Vernalization refers to the phenomenon 


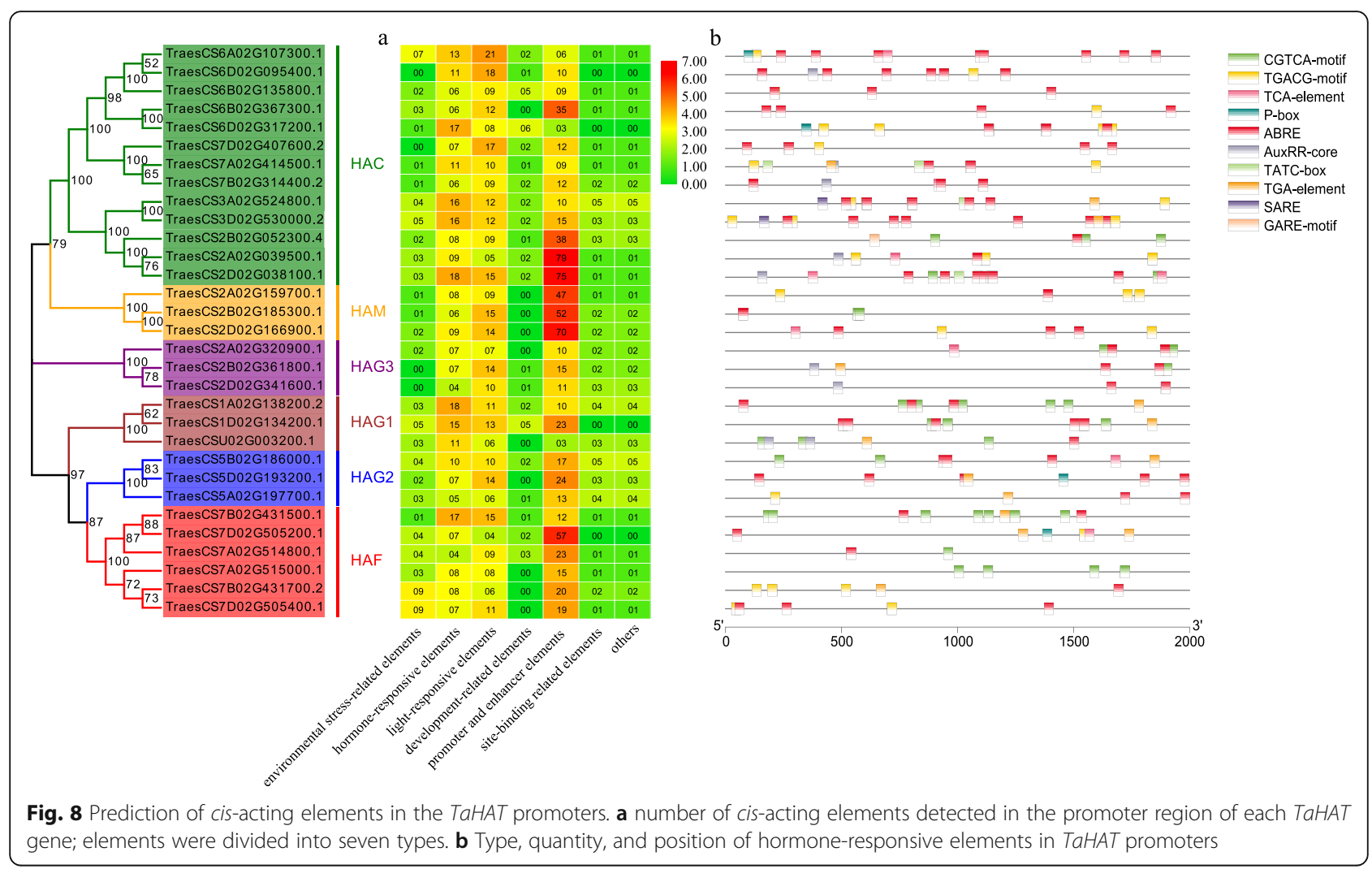

whereby plants must undergo a period of continuous low temperature before they can transition from vegetative to reproductive growth [51]. Further research on the relationship between vernalization and TaHATs may provide a new direction for the improvement of wheat yield. Although the function of HATs in response to cold exposure has been investigated, little is known about their potential role in response to viruses. After inoculation with BSMV, CWMV, or WYMV, the expression levels of TaHATs continued to increase from 7 to $16 \mathrm{dpi}$ in parallel with increased virus accumulation (Fig. 10). HAC is homologous to animal p300/CREB (cAMP-responsive element-binding protein)-binding proteins, participating in many physiological processes, including proliferation, differentiation and apoptosis [52]. Previous study has reported that HACs are involved in the ethylene signaling pathway [53]. It is well known that plant hormone plays multiple roles during the interaction of plant with virus. The expression of HAC in the wheat-BSMV interaction is higher than those of others. These result indicated that HAC may play different roles in response to different viral infection. It is worth noting that many wheat viruses infect plants at low temperatures. For example, the most suitable temperature for wheat streak mosaic virus (WSMV) infection is $15^{\circ} \mathrm{C}$ [54], the most suitable temperature for CWMV infection is $17^{\circ} \mathrm{C}$ [55]. Virus accumulation increases at low temperatures [56]. It is also interesting that WYMV and CWMV have hidden symptoms that leaves turn green at high temperatures of about $24{ }^{\circ} \mathrm{C}$ [57]. But BSMV has no hiding symptom of high temperature. Based on the results of our study, we can speculate that decreased acetylation levels at low temperature may predispose wheat to infection by various viruses in the field, whereas increased acetylation levels at high temperature may cause the hidden symptoms of WYMV and CWMV. Of course, these remain hypotheses at present. Further research will be required to verify the relationship between TaHATs, temperature, and viral infection, thereby laying a foundation for future research directions.

\section{Conclusions}

We identified 31 TaHATs and demonstrated that they could be divided into six groups. On the whole, members of the same groups were likely to have similar functions based on their similar structures and shared conserved motifs. The expression patterns of selected TaHATs differed among different tissues and at different temperatures. All of the measured TaHATs were upregulated after inoculation with BSMV, CWMV, and WYMV. These results indicate that TaHATs may be involved in wheat growth and development and play important roles in the response to stresses, including 


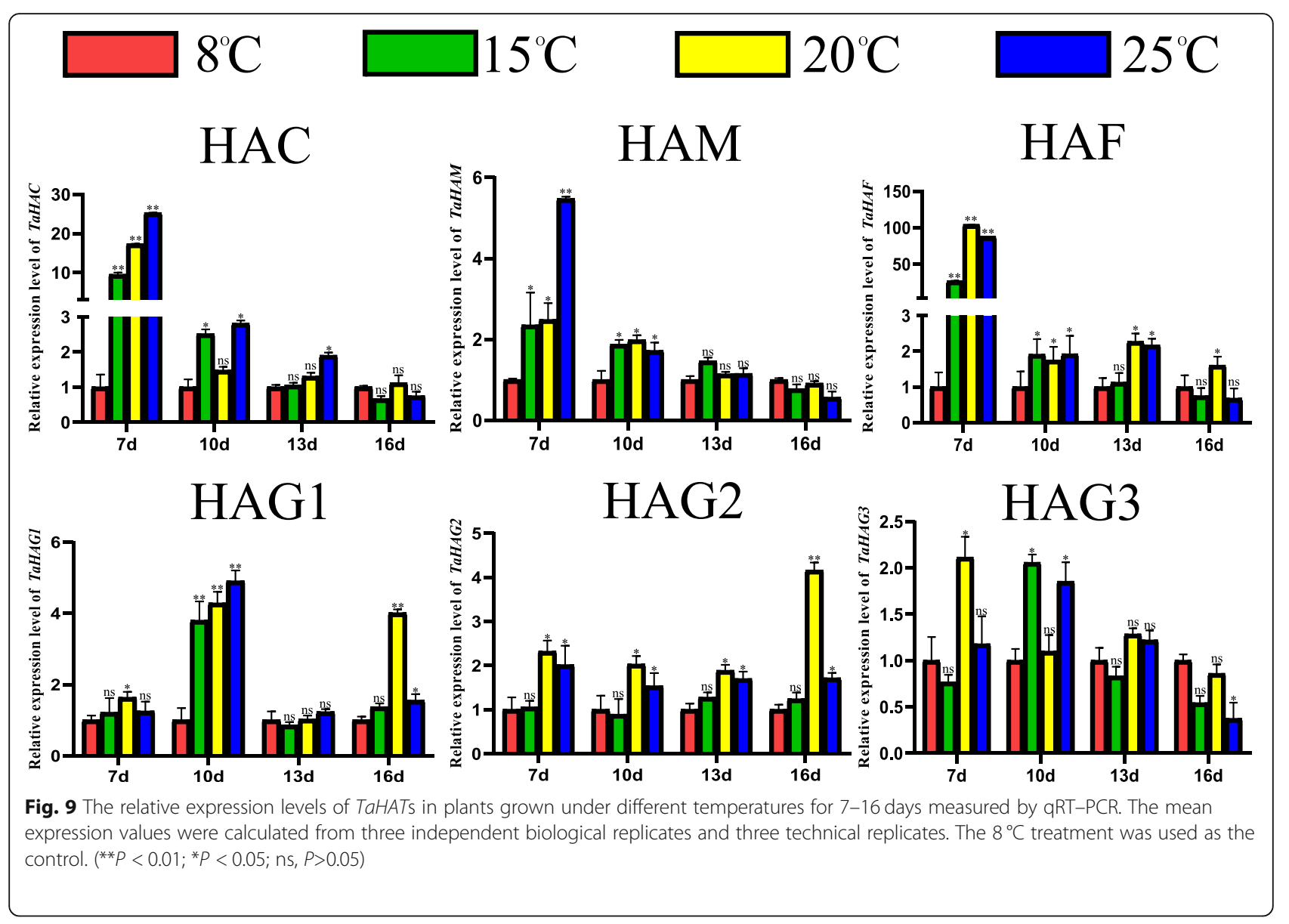

temperature extremes and viral infection. This work provides a basis for further functional characterization of TaHATs during wheat development and for exploring the relationship between $H A T \mathrm{~s}$, temperature, and viral infection in wheat.

\section{Methods}

Identification of the TaHAT family

Previously identified AtHAT and OsHAT protein sequences were downloaded from the Ensemble Plants database (http://plants.ensembl.org/index.html) [58] in order to identify all HAT proteins in $T$. aestivum using the AtHAT and OsHAT sequences as queries. Thirty-one putative TaHATs were identified through BLASTP searches $\left(\mathrm{E}<10^{-5}, \% \mathrm{ID}>50\right)$ performed against the Ensemble database. Next, AtHAT and OsHAT sequences were submitted to the Pfam database (http://pfam.xfam.org/) and the NCBI CD-search program (https://www.ncbi.nlm.nih.gov/ Structure/cdd/wrpsb.cgi) to obtain information on conserved protein domains in the HAT family $[59,60]$. We used the same method to analyze conserved domains in the 31 putative TaHATs and confirm that they belonged to the HAT family, and TBtools software was used for visualization [61]. Finally, detailed information on the
TaHATs, such as CDS, pI, and MW, were downloaded in batches from the Ensemble Plants database.

Multiple alignments and phylogenetic analysis

First, a multiple sequence alignment of AtHAT, OsHAT, and TaHAT protein sequences was imported into MEGA$\mathrm{X}$ and used to construct an unrooted phylogenetic tree with the neighbor-joining method and 1000 bootstrap replicates [62]. Finally, we used the online tool EVOLVIEW (https://evolgenius.info/evolview-v2/\#login) to create improved graphical presentations of the trees [63].

\section{Structure prediction of TaHATs protein}

We used SWISS-MODEL (https://swissmodel.expasy.org/) to make predictions for the structures of TaHATs protein [29]. Randomly select a gene from each group and different species for display: HAC (AT1G79000, LOC_Os06g49130, TraesCS6B02G135800.1), HAM (AT5G64610, LOC_Os07 g43360, TraesCS2D02G166900.1), HAF (AT3G19040, LO C_Os06g43790, TraesCS7A02G515000.1), HAG1 (AT3G 54610, LOC_Os10g28040, TraesCS1D02G134200.1), HAG2 (AT5G56740, LOC_Os09g17850, TraesCS5B02G186000.1), HAG3 (AT5G50320, LOC_Os04g40840, TraesCS2 D02G341600.1). 


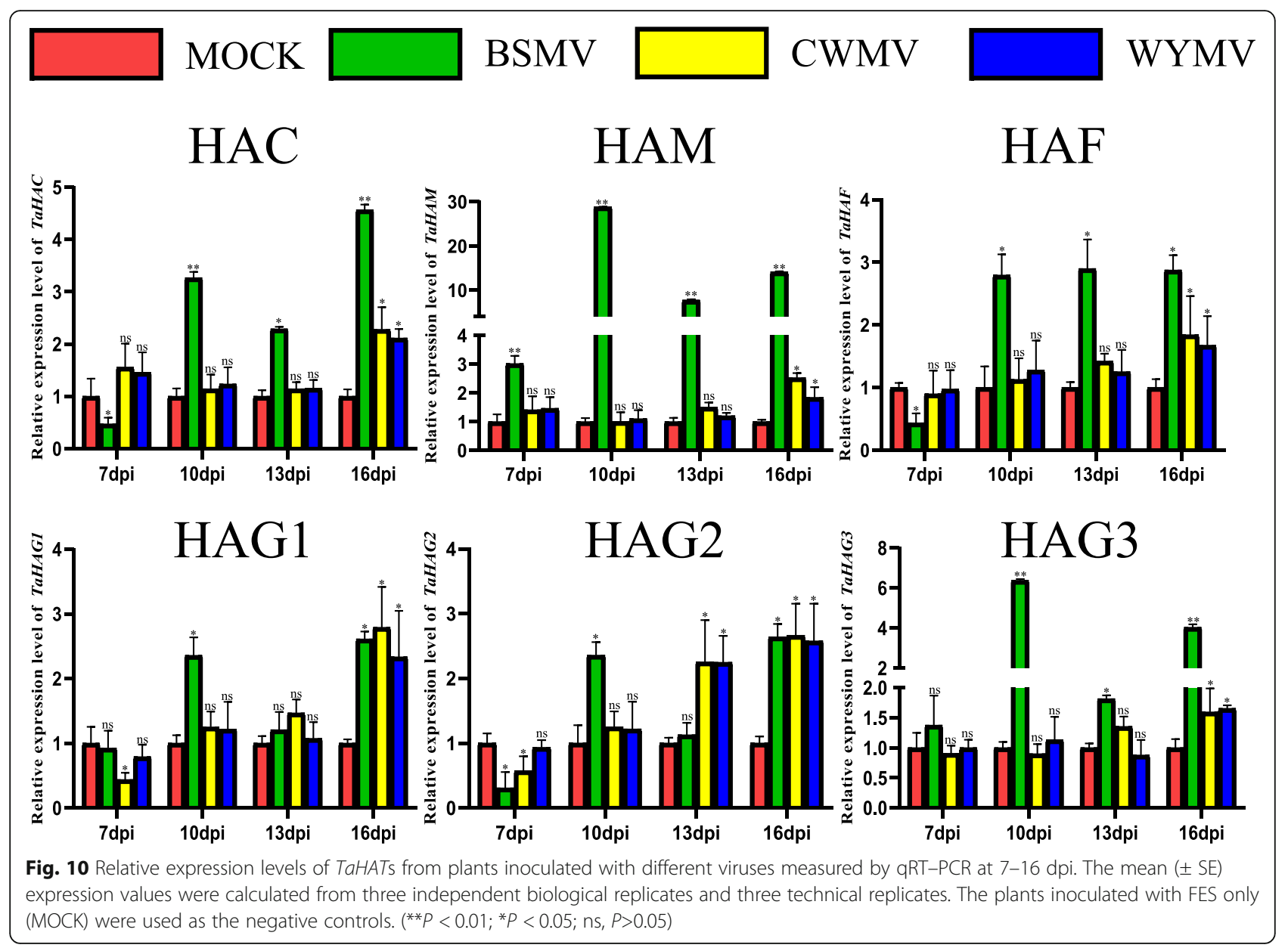

Gene structures and motif analysis

The genome annotation file (GTF) for $T$. aestivum was acquired from the Ensemble Plants database (http:// plants.ensembl.org/index.html) [58]. Gene structures were analyzed with TBtools Gene Structure View (Advanced) using the $T$. aestivum GTF file and the Newick Tree String of the TaHATs [61]. The Multiple Em for Motif Elicitation (MEME) online tool (http://alternate. meme-suite.org/tools/meme) was used to analyze protein motifs, with a maximum selection of 20 motifs [28].

\section{Chromosomal locations and Synteny analysis}

The initial TaHAT chromosomal positions and chromosome lengths were downloaded from the Ensemble Plants database (http://plants.ensembl.org/index.html), and MapChart software was used to visualize the distribution of TaHATs on chromosomes [58]. To investigate duplication of the TaHATs, we used TBtools to perform TaHAT synteny analysis [61].

\section{Calculation of $\mathrm{Ka} / \mathrm{Ks}$ values}

$\mathrm{The} \mathrm{Ka} / \mathrm{Ks}$ ratio of homologous gene pairs was used to determine whether they were under selection. $T a-T a$
(T. aestivum-T. aestivum) gene pairs were treated as paralogs and $\mathrm{Ta}-\mathrm{Os}$ ( $\mathrm{T}$. aestivum-O. sativa) pairs as orthologs. When $\mathrm{Ka} / \mathrm{Ks}$ is greater than one, genes are subject to positive selection. A $\mathrm{Ka} / \mathrm{Ks}$ of one implies neutral selection, and a $\mathrm{Ka} / \mathrm{Ks}$ less than one indicates purifying selection. TBtools software was used to calculate $\mathrm{Ka} / \mathrm{Ks}$ ratios and to estimate divergence times $(\mathrm{T})$ according to $\mathrm{T}=\mathrm{Ks} /\left(2 \times 9.1 \times 10^{-9}\right)$ Mya [33].

\section{Cis-acting elements in the TaHAT promoter regions}

The $2000 \mathrm{bp}$ upstream sequences of all TaHAT genes were extracted from the Ensemble Plants database in order to identify cis-elements in the putative promoter regions using PlantCARE software (http://bioinfor matics.psb.ugent.be/webtools/plantcare/html/) [64]. The identified cis-acting elements were classified by their different functions and visualized using TBtools software [61].

Plant materials, growth, and virus inoculation Yangmai 158 wheat was grown in a glasshouse at $23^{\circ} \mathrm{C}$ with a $16 \mathrm{~h}$ light, $8 \mathrm{~h}$ dark photoperiod. Stress treatments were applied when the wheat had reached the three-leaf 
stage. Three-leaf-stage wheat was then used to analyze gene expression profiles under temperature stress and viral inoculation and in five tissue types: top leaf (TL), middle leaf (ML), bottom leaf (BL), stem (ST) and roots (RO). RO tissue was treated as the control.

For temperature stress treatments, plants were placed at different temperatures $\left(8,15,20\right.$, and $\left.25^{\circ} \mathrm{C}\right)$ in growth cabinets with $16 \mathrm{~h}$ light $/ 8 \mathrm{~h}$ dark photoperiod. The plants placed under $8{ }^{\circ} \mathrm{C}$ were used as the controls.

For the virus inoculation treatments, plants were inoculated with barley streak mosaic virus (BSMV), Chinese wheat mosaic virus (CWMV), or wheat yellow mosaic virus (WYMV) through in vitro transcription and mechanical friction. BSMV-based gene vectors were kindly provided by Dr. Dawei Li, China [65]. CWMV-based and WYMV-based gene vectors were kindly provided by Dr. Jian Yang, China $[56,66]$. The three viruses have the same inoculation method, and we will take plants inoculated with BSMV as an example. First, plasmid transcripts of BSMV RNA $\alpha, \beta$, and $\gamma$ were linearized for in vitro transcription. Second, the linearized plasmids were mixed in a molar ratio of 1:1:1 with an equal amount of excess inoculation buffer (FES) $(0.06 \mathrm{M}$ potassium phosphate, $0.1 \mathrm{M}$ glycine, $1 \%$ bentonite, $1 \%$ sodium pyrophosphate decahydrate, $1 \%$ celite, $\mathrm{pH} 8.5)[65,66]$. Finally, the mixture was inoculated into leaves of threeleaf-stage wheat seedlings. The plants inoculated with FES only (MOCK) were used as negative controls.

\section{Total RNA extraction and quantitative real-time PCR analysis}

Total RNA was extracted using the TRIzol reagent (Invitrogen) following the manufacturer's instructions and stored at $-80^{\circ} \mathrm{C}$ until use. The first strand cDNA was synthesized using a First Strand cDNA Synthesis Kit (Toyobo, Kita-ku, Osaka, Japan) and $1 \mu \mathrm{g}$ total RNA per $20 \mu \mathrm{l}$ reaction. The qRT-PCR analysis was performed using an ABI7900HT Sequence Detection System (Applied Biosystems, Foster City, CA, USA) with Hieff qPCR SYBR Green Master Mix (Yeasen, Shanghai, China). At least three biological replicates and three technical replicates were used for all qPCR analyses in this study. The T. aestivum cell division cycle $(C D C)$ gene (accession number $\mathrm{XM}_{-}$ 020313450) was used as the internal reference gene to calculate relative gene expression levels using the $2^{-\triangle \Delta C(t)}$ method [66-68]. Primer sequences used in the qRT-PCR reactions are presented in Table S3.

\section{Supplementary Information}

The online version contains supplementary material available at https://doi. org/10.1186/s12864-020-07348-6.

Additional file 1: Table S1. List of HATS CDS and protein sequences from Arabidopsis thaliana, Oryza sativa, and Triticum aestivum
Additional file 2: Table S2. The MEME motif sequences and lengths of the TaHATS

Additional file 3: Table S3. Specific Primers used for this article. Additional file 4: Figure S1. Predicted structures of three TaHAC proteins. TaHAC is TraesCS6B02G135800.1

Additional file 5: Figure S2. Chromosome locations of TaHAT genes. Chromosomes are represented by cylinders, and homologous chromosomes are filled with the same color. The brown font represents the HAG1 group, the green font represents the HAC group, the orange font represents the HAM group, the purple font represents the HAG3 group, the blue font represents the HAG2 group, and the red font represents the HAF group

Additional file 6: Figure S3. Differential expression of representative TaHATs estimated by qRT-PCR (raw data) in different tissues: top leaf (TL), middle leaf (ML), bottom leaf (BL), stem (ST), and roots (RO). Mean expression values were calculated from three independent biological replicates and are expressed relative to that of roots

Additional file 7: Figure S4. Reverse transcription PCR (RT-PCR) detection of BSMV, CWMV and WYMV infections. Three plants were analyzed for each treatment. Total RNA from health wheat plant was used as a negative control (-). Diluted plasmid BSMV- $\beta$, CWMV RNA 2, WYMV RNA 1 were used as the positive control (+) for BSMV, CWMV and WYMV, respectively.

\section{Abbreviations}

HAT: Histone acetyltransferase; HADC: Histone deacetylase; BSMV: Barley streak mosaic virus; CWMV: Chinese wheat mosaic virus; WYMV: Wheat yellow mosaic virus; CDC: Cell division cycle; qRT-PCR: quantitative real-time PCR; WSMV: Wheat streak mosaic virus

\section{Acknowledgements}

We thank Dr. Dawei Li for providing the BSMV-based gene vectors.

\section{Authors' contributions}

GSQ, YJ and ZKL initiated and designed the experiments. GSQ, LLZ, HXL, JP,

$C L N, X M Z, L T T, Z T Y$ and ZF performed the experiments and collected the

data. GSQ analyzed the data and wrote the manuscript. LLZ, YJ and ZKL

revised the manuscript. All authors read and approved the final manuscript.

\section{Funding}

This work was funded by the National Key R\&D Plan in China (2018YFD0200507, 2017YFD-0201701, 2018YFD0200408), National Natural Science Foundation of China (31901954), Natural Science Foundation of Ningbo City (2019A610415, 2019A610410), Public Projects of Ningbo City (202002 N3004), National Key Project for Research on Transgenic Biology (2016ZX08002-001), China Agriculture Research System from the Ministry of Agriculture of the P.R. China (CARS-03), and K.C. Wong Magna Funding in Ningbo University.

\section{Availability of data and materials}

The data included in this article and the additional files are available. The sequences of Arabidopsis thaliana, Oryza sativa and Triticum aestivum are available in the Ensemble Plants database (http://plants.ensembl.org/index. $h$ tml).

Ethics approval and consent to participate

Not applicable.

\section{Consent for publication}

Not applicable.

\section{Competing interests}

The authors declare that they have no competing interests.

\section{Author details}

${ }^{1}$ State Key Laboratory for Managing Biotic and Chemical Threats to the Quality and Safety of Agro-products, Key Laboratory of Biotechnology in Plant Protection of Ministry of Agriculture and Zhejiang Province, Institute of Plant Virology, Ningbo University, Ningbo 315211, China. ${ }^{2}$ Yantai Academy of 
Agricultural Science, Yantai 265500, China. ${ }^{3}$ School of Life Sciences, Yantai University, Yantai 264005, China.

\section{Received: 12 October 2020 Accepted: 23 December 2020 Published online: 11 January 2021}

\section{References}

1. Liu XC, Yang SG, Zhao ML, Luo M, Yu C-W, Chen C-Y, Tai R, Wu KQ. Transcriptional repression by histone deacetylases in plants. Mol Plant. 2014; 7(5):764-72.

2. Yuan LY, Liu XC, Luo M, Yang SG, Wu KQ. Involvement of histone modifications in plant abiotic stress responses. J Integr Plant Biol. 2013; 55(10):892-901.

3. Pandey R, Muller A, Napoli CA, Selinger DA, Pikaard CS, Richards EJ, Bender J, Mount DW, Jorgensen RA. Analysis of histone acetyltransferase and histone deacetylase families of Arabidopsis thaliana suggests functional diversification of chromatin modification among multicellular eukaryotes. Nucleic Acids Res. 2002;30(23):5036-55.

4. Struhl K. Histone acetylation and transcriptional regulatory mechanisms. Genes Dev. 1998;12(5):599-606.

5. Eberharter A, Becker PB. Histone acetylation: a switch between repressive and permissive chromatin - second in review series on chromatin dynamics. EMBO Rep. 2002;3(3):224-9.

6. Sterner DE, Berger SL. Acetylation of histones and transcription-related factors. Microbiol Mol Biol Rev. 2000;64(2):435-59.

7. Kuo M-H, Allis CD. Roles of histone acetyltransferases and deacetylases in gene regulation. BioEssays. 1998;20(8):615-26.

8. Chen ZJ, Tian L. Roles of dynamic and reversible histone acetylation in plant development and polyploidy. Biochim Biophys Acta. 2007;1769(5-6):295307

9. Liu X, Yang S, Yu C-W, Chen C-Y, Wu K. Histone acetylation and plant development. Enzymes. 2016:40:173-99.

10. Liu X, Luo M, Zhang W, Zhao JH, Zhang JX, Wu KQ, Tian LN, Duan J. Histone acetyltransferases in rice (Oryza sativa L.): phylogenetic analysis, subcellular localization and expression. BMC Plant Biol. 2012;12:145.

11. Papaefthimiou D, Likotrafiti E, Kapazoglou A, Bladenopoulos K, Tsaftaris A. Epigenetic chromatin modifiers in barley: III. Isolation and characterization of the barley GNAT-MYST family of histone acetyltransferases and responses to exogenous ABA. Plant Physiol Biochem. 2010;48(2-3):98-107.

12. Aquea F, Timmermann T, Arce-Johnson P. Analysis of histone acetyltransferase and deacetylase families of Vitis vinifera. Plant Physiol Biochem. 2010:48(2-3):194-9.

13. Cigliano RA, Sanseverino W, Cremona G, Ercolano MR, Conicella C, Consiglio FM. Genome-wide analysis of histone modifiers in tomato: gaining an insight into their developmental roles. BMC Genomics. 2013;14:57

14. Peng MJ, Ying PY, Liu XC, Li CQ, Xia R, Li JG, Zhao ML. Genome-wide identification of histone modifiers and their expression patterns during fruit abscission in litchi. Front Plant Sci. 2017:8:639.

15. Georgieva El, Lopez-Rodas G, Sendra R, Grobner P, Loidl P. Histone acetylation in Zea mays. II. Biological significance of post-translational histone acetylation during embryo germination. J Biol Chem. 1991;266(28): 18751-60.

16. Latrasse D, Benhamed M, Henry Y, Domenichini S, Kim W, Zhou D-X, Delarue M. The MYST histone acetyltransferases are essential for gametophyte development in Arabidopsis. BMC Plant Biol. 2008;8:121.

17. Kornet N, Scheres B. Members of the GCN5 histone acetyltransferase complex regulate PLETHORA-mediated root stem cell niche maintenance and transit amplifying cell proliferation in Arabidopsis. Plant Cell. 2009;21(4): 1070-9.

18. Benhamed M, Bertrand C, Servet C, Zhou D-X. Arabidopsis GCN5, HD1, and TAF1/HAF2 interact to regulate histone acetylation required for lightresponsive gene expression. Plant Cell. 2006;18(11):2893-903.

19. Bertrand C, Benhamed M, Li Y-F, Ayadi M, Lemonnier G, Renou J-P, Delarue M, Zhou D-X. Arabidopsis HAF2 gene encoding TATA-binding protein (TBP)associated factor TAF1, is required to integrate light signals to regulate gene expression and growth. J Biol Chem. 2005;280(2):1465-73.

20. Deng WW, Liu CY, Pei YX, Deng X, Niu LF, Cao XF. Involvement of the histone acetyltransferase AtHAC1 in the regulation of flowering time via repression of flower locus in Arabidopsis. Plant Physiol. 2007;143(4):1660-8.

21. Han S-K, Song J-D, Noh Y-S, Noh B. Role of plant CBP/p300-like genes in the regulation of flowering time. Plant J. 2006;49(1):103-14
22. Brunet A, Sweeney LB, Sturgill JF, Chua KF, Greer PL, Lin YX, Tran H, Ross SE, Mostoslavsky R, Cohen HY, et al. Stress-dependent regulation of FOXO transcription factors by the SIRT1 deacetylase. Science. 2004;303(5666):20115.

23. Mao YP, Pavangadkar KA, Thomashow MF, Triezenberg SJ. Physical and functional interactions of Arabidopsis ADA2 transcriptional coactivator proteins with the acetyltransferase GCN5 and with the cold-induced transcription factor CBF1. Biochim Biophys Acta. 2006;1759(1-2):69-79.

24. Yin ZY, Chen C, Yang J, Feng WZ, Liu XY, Zuo RF, Wang JZ, Yang LN, Zhong $\mathrm{KL}$, Gao CY, et al. Histone acetyltransferase MoHat1 acetylates autophagyrelated proteins MoAtg3 and MoAtg9 to orchestrate functional appressorium formation and pathogenicity in Magnaporthe oryzae. Autophagy. 2019:15(7):1234-57.

25. Zhao W, Wang T, Liu SS, Chen QQ, Qi RD. The histone acetyltransferase PsGcn5 mediates oxidative stress responses and is required for full virulence of Phytophthora sojae. Microb Pathog. 2015;87:51-8.

26. Andika IB, Jamal A, Kondo H, Suzuki N. SAGA complex mediates the transcriptional up-regulation of antiviral RNA silencing. Proc Natl Acad Sci U S A. 2017;114(17):E3499-506.

27. Chen L, Zhao JQ, Song JC, Jameson PE. Cytokinin dehydrogenase: a genetic target for yield improvement in wheat. Plant Biotechnol J. 2020;18(3):61430 .

28. Wang Y, Pan F, Chen DM, Chu WY, Liu HL, Xiang Y. Genome-wide identification and analysis of the Populus trichocarpa TIFY gene family. Plan Physiol Biochem. 2017;115:360-71.

29. Waterhouse A, Bertoni M, Bienert S, Studer G, Tauriello G, Gumienny R, Heer FT, de Beer TAP, Rempfer C, Bordoli L, et al. SWISS-MODEL: homology modelling of protein structures and complexes. Nucleic Acids Res. 2018; 46(W1):W296-303

30. Shiu S-H, Bleecker AB. Expansion of the receptor-like kinase/Pelle gene family and receptor-like proteins in Arabidopsis. Plant Physiol. 2003;132(2): 530-43.

31. Dhar J, Chakrabarti P. Structural motif, topi and its role in protein function and fibrillation. Mol Omics. 2018;14(4):247-56

32. Knox AK, Dhillon T, Cheng HM, Tondelli A, Pecchioni N, Stockinger EJ. CBF gene copy number variation at frost Resistance-2 is associated with levels of freezing tolerance in temperate-climate cereals. Theor Appl Genet. 2010; 121(1):21-35.

33. Hurst LD. The Ka/Ks ratio: diagnosing the form of sequence evolution Trends Genet. 2002;18(9):486.

34. Li ZY, Li G, Cai MX, Priyadarshani S, Aslam M, Zhou Q, Huang XY, Wang XM, Liu YQ, Qin Y. Genome-wide analysis of the YABBY transcription factor family in pineapple and functional identification of AcYABBY4 involvement in salt stress. Int J Mol Sci. 2019;20(23):5863.

35. Mai A, Rotili D, Tarantino D, Nebbioso A, Castellano S, Sbardella G, Tini M, Altucci L. Identification of 4-hydroxyquinolines inhibitors of p300/CBP histone acetyltransferases. Bioorg Med Chem Lett. 2009:19(4):1132-5.

36. Kikuchi H, Nakayama T. GCN5 and BCR signalling collaborate to induce premature B cell apoptosis through depletion of ICAD and IAP2 and activation of caspase activities. Gene. 2008;419(1-2):48-55.

37. Boycheva I, Vassileva V, lantcheva A. Histone acetyltransferases in plant development and plasticity. Curr Genomics. 2014;15(1):28-37.

38. Wang Z, Cao H, Chen FY, Liu YX. The roles of histone acetylation in seed performance and plant development. Plant Physiol Biochem. 2014;84:12533.

39. Mou YF, Liu YY, Tian SJ, Guo QP, Wang CS, Wen SS. Genome-wide identification and characterization of the OPR gene family in wheat (Triticum aestivum L.). Int J Mol Sci. 2019;20(8):1914.

40. Zhao Y, Ma RY, Xu DL, Bi HH, Xia ZL, Peng HR. Genome-wide identification and analysis of the AP2 transcription factor gene family in wheat (Triticum aestivum L.). Front Plant Sci. 2019;10:1286.

41. Das M, Haberer G, Panda A, Das Laha S, Ghosh TC, Schaffner AR. Expression pattern similarities support the prediction of orthologs retaining common functions after gene duplication events. Plant Physiol. 2016;171(4):2343-57.

42. Bharti K, Von Koskull-Doring P, Bharti S, Kumar P, Tintschl-Korbitzer A,

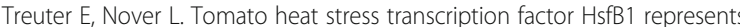
a novel type of general transcription coactivator with a histone-like motif interacting with the plant CREB binding protein ortholog HAC1. Plant Cell. 2004;16(6):1521-35.

43. Nelissen H, Fleury D, Bruno L, Robles P, De Veylder L, Traas J, Micol JL, Van Montagu M, Inze D, Van Lijsebettens M. The elongata mutants identify a 
functional elongator complex in plants with a role in cell proliferation during organ growth. Proc Natl Acad Sci U S A. 2005;102(21):7754-9.

44. Crane YM, Gelvin SB. RNAi-mediated gene silencing reveals involvement of Arabidopsis chromatin-related genes in agrobacterium-mediated root transformation. Proc Natl Acad Sci U S A. 2007;104(38):15156-61.

45. Pavangadkar K, Thomashow MF, Triezenberg SJ. Histone dynamics and roles of histone acetyltransferases during cold-induced gene regulation in Arabidopsis. Plant Mol Biol. 2010;74(1-2):183-200.

46. Servet C, Silva NC, Zhou DX. Histone acetyltransferase AtGCN5/HAG1 is a versatile regulator of developmental and inducible gene expression in Arabidopsis. Mol Plant. 2010;3(4):670-7.

47. Earley KW, Shook MS, Brower-Toland B, Hicks L, Pikaard CS. In vitro specificities of Arabidopsis co-activator histone acetyltransferases: implications for histone hyperacetylation in gene activation. Plant J. 2007; 52(4):615-26.

48. Chen ZZ, Zhang HR, Jablonowski D, Zhou XF, Ren XZ, Hong XH, Schaffrath $\mathrm{R}$, Zhu J-K, Gong ZZ. Mutations in ABO1/ELO2, a subunit of holo-Elongator, increase abscisic acid sensitivity and drought tolerance in Arabidopsis thaliana. Mol Cell Biol. 2006;26(18):6902-12.

49. Fina JP, Casati P. HAG3, a histone acetyltransferase, affects UV-B responses by negatively regulating the expression of DNA eepair enzymes and sunscreen content in Arabidopsis thaliana. Plant Cell Physiol. 2015;56(7): 1388-400.

50. Ramirez-Parra E, Frundt C, Gutierrez C. A genome-wide identification of E2Fregulated genes in Arabidopsis. Plant J. 2003;33(4):801-11.

51. Finnegan EJ. Vernalization. Curr Biol. 2012;22(12):R471-2.

52. Roelfsema JH, White SJ, Ariyurek Y, Bartholdi D, Niedrist D, Papadia F, Bacino CA, den Dunnen JT, van Ommen GJB, Breuning MH, et al. Genetic heterogeneity in Rubinstein-Taybi syndrome: mutations in both the CBP and EP300 genes cause disease. Am J Hum Genet. 2005;76(4):572-80.

53. Li C, Xu J, Li J, Li QY, Yang HC. Involvement of Arabidopsis histone acetyltransferase HAC family genes in the ethylene signaling pathway. Plant Cell Physiol. 2014;55(2):426-35.

54. Ranabhat NB, Seipel T, Lehnhoff EA, Miller ZJ, Owen KE, Menalled FD, Burrows ME. Temperature and alternative hosts influence aceria tosichella infestation and wheat streak mosaic virus infection. Plant Dis. 2018;102(3): 546-51.

55. Yang J, Zhang F, Xie L, Song X-J, Li J, Chen J-P, Zhang H-M. Functional identification of two minor capsid proteins from Chinese wheat mosaic virus using its infectious full-length cDNA clones. J Gen Virol. 2016:97(9): 2441-50.

56. Yang J, Zhang T-Y, Liao Q-S, He L, Li J, Zhang H-M, Chen X, Li J, Yang J, Li J$B$, et al. Chinese wheat mosaic virus-induced gene silencing in monocots and dicots at low temperature. Front Plant Sci. 2018;9:1627.

57. Andika IB, Sun LY, Xiang R, Li JM, Chen JP. Root-specific role for Nicotiana benthamiana RDR6 in the inhibition of Chinese wheat mosaic virus accumulation at higher temperatures. Mol Plant-Microbe Interact. 2013; 26(10):1165-75.

58. Bolser DM, Kerhornou A, Walts B, Kersey P. Triticeae resources in Ensembl Plants. Plant Cell Physiol. 2015;56(1):e3.

59. Finn RD, Bateman A, Clements J, Coggill P, Eberhardt RY, Eddy SR, Heger A, Hetherington K, Holm L, Mistry J, et al. Pfam: the protein families database. Nucleic Acids Res. 2014;42(Database issue):D222-30.

60. Marchler-Bauer A, Bryant SH. CD-Search: protein domain annotations on the fly. Nucleic Acids Res. 2004;32(Web Server issue):W327-31.

61. Chen CJ, Chen H, Zhang Y, Thomas HR, Frank MH, He YH, Xia R. TBtools: an integrative toolkit developed for interactive analyses of big biological data. Mol Plant. 2020;13(8):1194-202.

62. Zhou YZ, Zhang SH, Chen JH, Wan CS, Zhao W, Zhang B. Analysis of variation and evolution of SARS-CoV-2 genome. J South Med Univ. 2020; 40(2): $152-8$

63. Subramanian B, Gao SH, Lercher MJ, Hu SN, Chen W-H. Evolview v3: a webserver for visualization, annotation, and management of phylogenetic trees. Nucleic Acids Res. 2019:47(W1):W270-5.

64. Lescot M, Dehais P, Thijs G, Marchal K, Moreau Y, Van de Peer Y, Rouze P, Rombauts $\mathrm{S}$. PlantCARE, a database of plant cis-acting regulatory elements and a portal to tools for in silico analysis of promoter sequences. Nucleic Acids Res. 2002;30(1):325-7.

65. Kong JH, Chen WW, Shen JJ, Qin C, Lai TF, Zhang PC, Wang Y, Wu CQ, Yang $X$, Hong YG. Virus-induced gene complementation in tomato. Plant Signal Behav. 2013;8(11):e27142.
66. Zhang TY, Liu P, Zhong KL, Zhang F, Xu MZ, He L, Jin P, Chen JP, Yang J. Wheat yellow mosaic virus $\mathrm{Nlb}$ interacting with host light induced protein (LIP) facilitates its infection through perturbing the abscisic acid pathway in wheat. Biology (Basel). 2019;8(4):80.

67. Livak KJ, Schmittgen TD. Analysis of relative gene expression data using real-time quantitative PCR and the $2^{-\Delta \Delta C}$ T method. Methods. 2001;25(4): 402-8.

68. He L, Chen X, Yang J, Zhang TY, Li J, Zhang SB, Zhong KL, Zhang HM, Chen $J P$, Yang J. Rice black-streaked dwarf virus-encoded P5-1 regulates the ubiquitination activity of SCF E3 ligases and inhibits jasmonate signaling to benefit its infection in rice. New Phytol. 2020;225(2):896-912.

\section{Publisher's Note}

Springer Nature remains neutral with regard to jurisdictional claims in published maps and institutional affiliations.

\section{Ready to submit your research? Choose BMC and benefit from:}

- fast, convenient online submission

- thorough peer review by experienced researchers in your field

- rapid publication on acceptance

- support for research data, including large and complex data types

- gold Open Access which fosters wider collaboration and increased citations

- maximum visibility for your research: over $100 \mathrm{M}$ website views per year

At BMC, research is always in progress.

Learn more biomedcentral.com/submissions 